\title{
NITROGÊNIO E BORO NA PRODUÇÃO E INCIDÊNCIA DE HASTE OCA EM COUVE-FLOR 'SHARON' ( $\left.{ }^{1}\right)$
}

\author{
SIMONE DA COSTA MELLO $\left({ }^{*}\right)$; MÔNICA SARTORI DE CAMARGO $\left({ }^{3}\right)$; RAFAEL VIVIAN $\left({ }^{2}\right)$; \\ THAISA SAMPAIO NASCIMENTO $\left({ }^{2}\right)$; ÉRICA SANTOS DE OLIVEIRA $\left({ }^{2}\right)$; RAPHAEL BERTANHA $\left({ }^{2}\right)$
}

\begin{abstract}
RESUMO
Objetivou-se avaliar a influência de doses de nitrogênio (100, 150, 200 e $\left.250 \mathrm{~kg} \mathrm{ha}^{-1}\right)$ e de boro (0; $1,5 \mathrm{~kg} \mathrm{ha}^{-1}$ no plantio e $1,5 \mathrm{~kg} \mathrm{ha}^{-1}$ aos 45 dias após o transplante das mudas ; 3,0 kg ha $\mathrm{kg}^{-1}$ nolantio) na produtividade e na ocorrência de haste oca em couve-flor 'Sharon', sob blocos ao acaso, esquema fatorial, com quatro repetições. O experimento foi realizado em Piracicaba (SP), em condições de campo, em Nitossolo Vermelho Eutrófico, de novembro de 2005 a março de 2006. O nitrogênio e o boro não influenciaram a produtividade total e comercial, a massa da inflorescência, os teores de $\mathrm{N}$ e de $\mathrm{B}$ na folha e na inflorescência. A incidência de haste oca foi reduzida com a aplicação de B.
\end{abstract}

Palavras-chave: Brassica oleracea var. botrytis, adubação, deficiência, nutrição.

\section{ABSTRACT \\ NITROGEN AND BORON ON YIELD AND INCIDENCE OF HOLLOW STEM DISORDER IN CAULIFLOWER 'SHARON'}

\begin{abstract}
The objective was evaluate nitrogen $\left(100,150,200\right.$ and $\left.250 \mathrm{~kg} \mathrm{ha}^{-1}\right)$ and boron rates $\left(0 ; 1.5 \mathrm{~kg} \mathrm{ha}^{-1}\right.$ at planting and $1.5 \mathrm{~kg} \mathrm{ha}^{-1}$ after 45 transplanting days and $3.0 \mathrm{~kg} \mathrm{ha}^{-1}$ at planting) on yield and hollow stem disorder of cauliflower 'Sharon' in blocks randomized in factorial design with four replications. The experiment was carried out in field conditions, in Piracicaba, São Paulo State, at Nitossolo Vermelho Eutrófico soil during November/2005 until March/2006. The nitrogen and boron did not effect on commercial and total yield, inflorescence mass, N and B levels of leaves and inflorescences. Hollow stem incidence was reduced by B application.
\end{abstract}

Key words: Brassica oleracea var. botrytis, fertilization, deficiency; nutrition.

( $\left.{ }^{1}\right)$ Recebido para publicação em 20 de fevereiro de 2008 e aceito para publicação em 18 de fevereiro de 2009.

( $\left.{ }^{1}\right)$ Escola Superior de Agricultura Luiz de Queiroz/USP, Caixa Postal 09, 13418-900 Piracicaba (SP). E-mail: scmello@esalq.usp.br $\left(^{*}\right)$ Autor correspondente.

( $\left.{ }^{3}\right)$ Polo Regional Centro-Sul/APTA, Rodovia SP-127, km 30, Caixa Postal 28, 13412-050 Piracicaba (SP). E-mail:mscamarg@yahoo.com.br. 
A qualidade e a produtividade da couve-flor (Brassica oleracea var. botrytis) são fortemente influenciadas pelas condições climáticas e pelo manejo da adubação. Dentre os nutrientes, o boro (B) merece destaque pela alta exigência dessa hortaliça, podendo sua deficiência acarretar sérios prejuízos econômicos (GUPTA, 1993).

No Brasil, é comum a carência de B nos solos, principalmente nos arenosos e pobres em matéria orgânica (Malavolta et al. 1997). Em couve-flor, os sintomas de deficiência desse elemento são caracterizados pelo aparecimento de cavidade na haste principal e por pontuações escuras nas inflorescências (SHELP et al., 1992).

Esses efeitos estão relacionados à atividade do B em vários processos, entre eles a divisão e o alongamento celular (SHELP, 1993). Entretanto, a deficiência de B em couve-flor pode ocorrer mesmo em solo com teor de B considerado elevado (SHELP et al.,1995), principalmente quando essa hortaliça é cultivada no verão (SCAIFE e WURR, 1990) e em áreas intensamente adubadas com nitrogênio, que favorecem o crescimento mais acelerado das plantas (EvERAARTS, 2000).

Estudos sobre a ação conjunta do B e do N na incidência de haste oca em couve-flor revelam resultados contraditórios. Vigier e CUTCLIFFe (1984) não verificaram ocorrência desse distúrbio fisiológico para couve-flor cultivada com doses de até $90 \mathrm{~kg} \mathrm{ha}^{-1}$ de $\mathrm{N}$ e com 2 a $4 \mathrm{~kg} \mathrm{ha}^{-1}$ de B. Por outro lado, Everaarts e Putter (2003), utilizando doses mais altas de nitrogênio $\left(200 \mathrm{~kg} \mathrm{ha}^{-1}\right)$, verificaram aumento na incidência de haste oca, enquanto a aplicação de 2 $\mathrm{kg} \mathrm{ha}^{-1}$ de B não afetou essa característica. BATAL et al. (1997) observaram redução na ocorrência de haste oca em couve-flor com $269 \mathrm{~kg} \mathrm{ha}^{-1}$ de $\mathrm{N}$ e $8,8 \mathrm{~kg} \mathrm{ha}^{-1}$ de $\mathrm{B}$, em solos com 0,07 e 0,21 mg kg-1 de B.

Este trabalho foi realizado considerando a necessidade de maior conhecimento sobre a interação do $\mathrm{N}$ e do B na ocorrência de haste oca em couve-flor.

O experimento foi instalado em Piracicaba (SP), durante o período de 29 de novembro de 2005 a 4 de março de 2006. Foi utilizado o cultivar Sharon de couve-flor em função da sua adaptabilidade à região.

O solo da área experimental foi Nitossolo Vermelho Eutrófico A moderado textura argilosa, com as seguintes características: matéria orgânica $=31 \mathrm{~g}$ $\mathrm{dm}^{-3} ; \mathrm{P}($ resina $)=312 \mathrm{mg} \mathrm{dm}^{-3} ; \mathrm{K}, \mathrm{Ca}$ e $\mathrm{Mg}=15,6 ; 89,0$ e $36 \mathrm{mmol}_{\mathrm{c}} \mathrm{dm}^{-3}$, respectivamente; $\mathrm{S}=90 \mathrm{mg} \mathrm{dm}^{-3} ; \mathrm{pH}$ $\left(\mathrm{CaCl}_{2}\right)=6,1 ; \mathrm{CTC}=160,6 \mathrm{mmol}_{\mathrm{c}} \mathrm{dm}^{-3} ; \mathrm{V}=88 \%$ e B $=$ $0,84 \mathrm{mg} \mathrm{dm}^{-3}$.
O delineamento experimental foi o de blocos casualizados, com quatro repetições, distribuídos em arranjo fatorial $4 \times 3$, sendo quatro doses de N (100, 150,200 e $250 \mathrm{~kg} \mathrm{ha}^{-1}$ ) e três formas de aplicação de B $\left(0 ; 3,0 \mathrm{~kg} \mathrm{ha}^{-1}\right.$ no plantio e $1,5 \mathrm{~kg} \mathrm{ha}^{-1}$ no plantio e 1,5 $\mathrm{kg} \mathrm{ha}^{-1}$ em cobertura, aos 45 dias após o transplante das mudas). As aplicações de B foram feitas na forma de Boromol ( $8 \%$ de B e $0,8 \%$ de Mo) diretamente na cova, com posterior homogeneização do solo. Para a aplicação em cobertura, o adubo foi distribuído manualmente ao redor da haste da planta. As doses de $\mathrm{N}$ foram parceladas da seguinte forma: $60 \mathrm{~kg} \mathrm{ha}^{-1}$ de $\mathrm{N}$ no plantio e o restante, conforme o tratamento, em cobertura, sendo $25 \%$ aos 20 dias após o transplante das mudas (DAT), $25 \%$ aos 30 DAT e $50 \%$ aos 45 DAT. Para o N, utilizou-se o nitrato de amônio ( $32 \%$ de N). Foram aplicados também $200 \mathrm{~kg} \mathrm{ha}^{-1}$ de $\mathrm{P}_{2} \mathrm{O}_{5}$ e $120 \mathrm{~kg} \mathrm{ha}^{-1}$ de $\mathrm{K}_{2} \mathrm{O}$, nas formas de superfosfato simples $\left(18 \% \mathrm{P}_{2} \mathrm{O}_{5}\right)$ e cloreto de potássio $\left(60 \% \mathrm{~K}_{2} \mathrm{O}\right)$, conforme recomendações de TRANI et al. (1997).

As mudas foram produzidas em bandejas de poliestireno expandido contendo 200 células, as quais foram preenchidas com substrato à base de casca de pinus e mantidas em casa-de-vegetação até o transplante. Após 35 dias da semeadura, as mudas foram transferidas para canteiros de $36 \mathrm{~m}^{2}$, utilizandose o espaçamento de $1 \mathrm{~m}$ entrelinhas e $0,5 \mathrm{~m}$ entre plantas. Cada parcela foi composta por 12 plantas distribuídas em duas linhas de plantio.

A colheita teve início aos 61 DAT, estendendose até $4 / 3 / 2007$. Foram avaliados a ocorrência de haste oca, a massa da inflorescência, o teor de $\mathrm{N}$ e de $B$ nas inflorescências, a produtividade total e produtividade comercial (inflorescências sem a presença de sintomas de deficiência de B). Na terceira folha expandida no início do florescimento, avaliaramse também os teores de $\mathrm{B}$ e de $\mathrm{N}$. As análises químicas do $\mathrm{N}$ e do $\mathrm{B}$ foram realizadas através das digestões sulfúrica, descritas por Malavolta et al. (1997) e ABREU et al. (1994) respectivamente. Os dados obtidos foram submetidos à análise de variância pelo teste $F$, sendo avaliados os modos de aplicação e doses de B pelo teste Tukey e as doses de $\mathrm{N}$ pela análise de regressão, com o auxílio do programa SAS (SAS INSTITUTE, 1996).

Para o nitrogênio isoladamente, a análise de regressão não acusou efeito significativo sobre a massa fresca das inflorescências, produtividade total, produtividade comercial, teores de $\mathrm{B}$ e de $\mathrm{N}$ nas folhas e nas inflorescências da couve-flor 'Sharon'. Para EveraArts e Moel (1995), a ausência de resposta da couve-flor ao $\mathrm{N}$ foi em razão da elevada disponibilidade de $\mathrm{N}$ mineral observada em solos intensamente adubados com esse elemento, o que 
também pode ter ocorrido na área onde foi implantado o presente experimento, pelo fato de a mesma ser freqüentemente adubada com fertilizantes nitrogenados.

A massa fresca das inflorescências, produtividade total, produtividade comercial, teores de $\mathrm{B}$ e de $\mathrm{N}$ nas folhas e nas inflorescências da couveflor 'Sharon' também não foram afetadas pela aplicação isolada de B (Tabela 1). Resultados semelhantes foram conseguidos por BATAL et al. (1997), e cuja produção total e a massa das inflorescências de couve-flor não foram influenciadas pelas aplicações de 2,2; 4,4 e 8,8 $\mathrm{kg} \mathrm{ha}^{-1}$ de B, em solo com $0,21 \mathrm{mg} \mathrm{kg}^{-1}$ de B e alta CTC. Já em solo com baixo teor de B $\left(0,07 \mathrm{mg} \mathrm{dm}^{-3}\right)$ e CTC de $25,5 \mathrm{mmol}_{\mathrm{c}} \mathrm{dm}^{-3}$, os autores obtiveram aumento nos valores dessas características com a aplicação de $4,4 \mathrm{~kg} \mathrm{ha}^{-1}$ de B, atestando a influência da fertilidade do solo na resposta da planta à adição de boro. PizetTa et al. (2005) também tiveram resposta positiva quanto à produtividade de couve-flor produzida no inverno (30 $\mathrm{t} \mathrm{ha}^{-1}$ ) com 5,5 kg ha ${ }^{-1}$ de B em solo de textura arenosa. Dessa forma, pode-se afirmar que o teor alto de B $(0,84$ $\mathrm{mg} \mathrm{kg}^{-1}$ ) no solo foi responsável pela ausência de efeito desse nutriente na produção de couve-flor.

Por outro lado, a aplicação de $3 \mathrm{~kg} \mathrm{ha}^{-1} \mathrm{~B}$, seja no plantio, seja fracionado, reduziu a ocorrência de haste oca (Tabela 1). BATAL et al. (1997) obtiveram redução de haste oca em couve-flor com doses entre 2,2 e $8,8 \mathrm{~kg} \mathrm{ha}^{-1} \mathrm{de}$ B. CAMARGo et al. (2008) também observaram redução drástica no número de plantas com haste oca $(4,06 \%)$ com a aplicação de $3 \mathrm{~kg} \mathrm{ha}^{-1}$ de $\mathrm{B}$ em relação à testemunha, cujo valor verificado para essa anomalia foi de $46,87 \%$. Esse resultado indica a necessidade de suprimento de $B$, mesmo em solo argiloso com teor elevado desse elemento, para a melhoria da qualidade da couve-flor 'Sharon', em função do comportamento deste micronutriente na planta, da época de cultivo e das características genéticas da cultivar.

Em couve-flor, sabe-se que o B possui baixa mobilidade via floema, o que não garante a sua redistribuição das folhas para os órgãos de maior demanda que são as inflorescências (GUPTA, 1993). Associado a isso, o período de cultivo (verão), caracterizado por precipitação pluvial elevada, pode ter reduzido a disponibilidade de $\mathrm{B}$ por motivo desse elemento ser altamente móvel, consequentemente lixiviado do solo.

Esse fato faz com que a adubação com B garanta maior nível desse elemento nos tecidos onde sua deficiência se manifesta, contribuindo para a menor ocorrência de haste oca. Além disso, deve-se levar em consideração que a cultivar empregada é exigente em $\mathrm{B}$, o que ajuda a explicar a redução dessa anomalia com seu fornecimento.

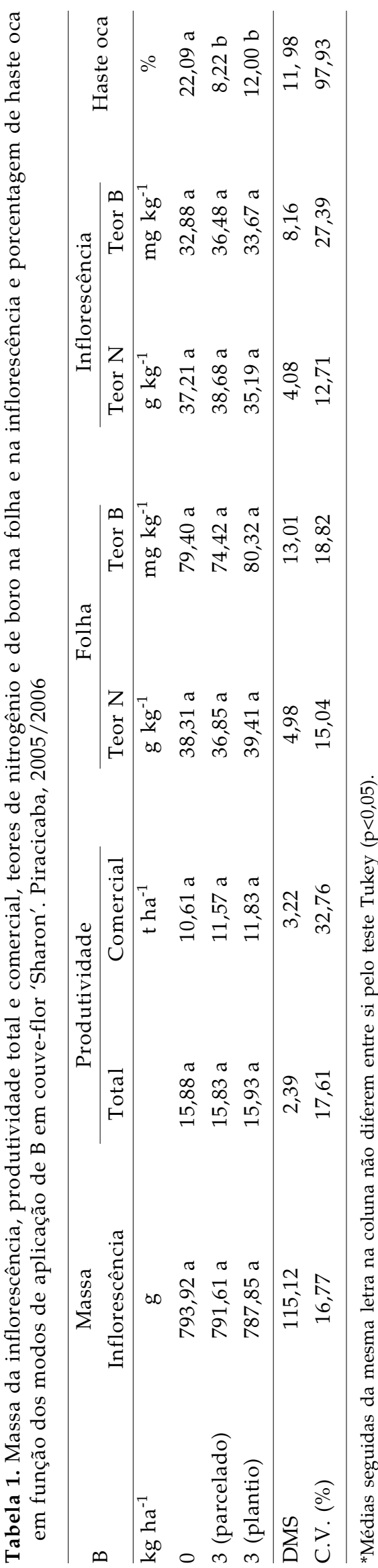

Bragantia, Campinas, v.68, n.3, p.761-764, 2009 
A interação entre as doses de $\mathrm{N}$ e os modos de aplicação de B não foi significativa sobre as características avaliadas.

O mesmo foi observado por BATAL et al. (1997), em que a adubação nitrogenada (269 $\mathrm{kg} \mathrm{ha}^{-1}$ de N) não influenciou a ocorrência de haste oca na presença de B (2,2 a 8,8 $\mathrm{kg} \mathrm{ha}^{-1}$ de B) em plantas de couve-flor. Interação significativa, por outro lado, ocorreu para a produção de couve-flor, cujo valor máximo foi obtido com a aplicação de $200 \mathrm{~kg} \mathrm{ha}^{-1}$ de $\mathrm{N}$ na taxa de $0,125 \%$ de B (Kotur, 1997).

A ocorrência de haste oca também não foi influenciada pelo nitrogênio, concordando com os resultados observados por BATAL et al. (1997). Entretanto, Scaife e WurR (1990), Kotur (1997) e EveraArts e PUtTer (2003) constataram aumento na incidência dessa anomalia, utilizando doses semelhantes às utilizadas no presente experimento. Segundo Vigier e Cutcliffe (1984), EveraArts (2000) e BÈLEC et al. (2001), doses elevadas de $\mathrm{N}$ aumentaram a incidência de haste oca em couve-flor pelo fato de a absorção do $B$ não ter ocorrido na mesma velocidade que a taxa de crescimento da planta, sendo acelerada pela adubação nitrogenada.

Mesmo em solo com alto teor de B $\left(0,84 \mathrm{mg} \mathrm{dm}^{-3}\right)$, a aplicação do elemento foi necessária para redução da incidência de haste oca em couve-flor 'Sharon'.

\section{REFERÊNCIAS}

ABREU, C.A.; ABREU, M.F.; RAIJ, B.van; BATAGLIA,O.; ANDRADE, J.C. Extraction of boron from soil by microwave heating for ICP-AES determination. Communications in Soil Science and Plant Analysis, v.25, p.3321-3333,1994.

BABIK, I.; RUMPEL, J.; ELKNER, K. The influence of nitrogen fertilization on yield, quality a senescence of Brussels sprouts. Acta Horticulturae, v.404, p.353-359, 1994.

BATAL, K.M.; GRANBERRY, D.M.; MULLINIX JR., B.G. Nitrogen, magnesium and boron applications affect cauliflower yield, curd mass and hollow stem disorder. HortScience, v.32, p.75-78, 1997.

BÉLEC, C.; VILLENEUVE, S.; COULOMBE, J.; TREMBLAY, N. Influence of nitrogen fertilization on yield, hollow stem incidence and sap nitrate concentration in broccoli. Canadian Journal of Plant Science, v.81, p.765-772, 2001.

BRACY, P.R.; PARISH, R.L.; BERGERON, P.E. Sidress N application methods for broccoli production. Journal of Vegetable Crop Production, v.1, p.63-71, 1995.

CAMARGO, M.S.; MELLO, S.C.; FOLTRAN, D. E.; CARMELLO, Q.A.C. Produtividade e podridão parda em couve-flor de inverno influenciadas pelo nitrogênio e boro. Bragantia, v.67, p.371-375, 2008.
EVERAARTS, A.P. Nitrogen balance during growth of cauliflower. Science Horticulturae, n.83, p.173-186, 2000.

EVERAARTS, A.P.; MOEL, C.P.de. The effect of nitrogen and the method of application on the yield of cauliflower. Netherlands Journal of Agricultural Science, v.43, n.4, p.409-418, 1995.

EVERAARTS, A.P.; PUTTER, H. de. Hollow stem in cauliflower. Acta Horticulturae, n.607, p.187-190, 2003.

GUPTA, U. C. Responses to boron on field and horticultural crop yields. In: GUPTA, U.C. (Ed.). Boron and its role in crop production. Boca Raton: CRC Press, 1993. p. 177-185.

KOTUR, S.C. Nitrogen-boron interaction in cauliflower (Brassica oleraceae var. botrytis L.) on an Alfisol. Journal of the Indian Society of Soil Science, v.45, p.519-522, 1997.

MALAVOLTA, E.; VITTI, G.C.; OLIVEIRA, S.A. Avaliação do estado nutricional das plantas: princípios e aplicações. 2.ed. Piracicaba: Potafós, 1997, p.231-305.

PIZETTA, L.C.; FERREIRA, M.E.; CRUZ, M.C.P.; BARBOSA, J.C. Resposta de brócolis, couve-flor e repolho à adubação com boro em solo arenoso. Horticultura Brasileira, Brasília, v.23, n.2, p.51-56, 2005.

SAS Institute INC. The SAS-system for windows : release 6.11(software). Cary: SAS Institute. 1996.

SCAIFE, A.; WURR, D.C.E. Effects of nitrogen and irrigation on hollow stem of cauliflower (Brassica oleraceae var botrytis). Journal of Horticultural Science, v.65, n. 1, p. 25-29, 1990.

SHELP, B.J. et al. Boron mobility in plants. Physilogoly Plantarum, v.94, p.356-361, 1995.

SHELP, B.J. Physiology and biochemistry of boron in plants. In: GUPTA, U.C. (Ed.). Boron and its role in crop production. Boca Raton: CRC Press, 1993. p. 53-85.

SHELP, B.J.; SHATTUCK, V.I.; McLELLAN, D.; LIU, L. Boron nutrition and the composition of glucosinolates and soluble nitrogen compounds in two broccoli (Brassica oleraceae var. italica) cultivars. Canadian Journal Plant Science, v. 72, p. 889-899, 1992.

TRANI, P.E.; PASSOS, F.A.; AZEVEDO, J.A.; TAVARES, M. Brócolos, couve-flor e repolho. In: RAIJ, B.van; CANTARELLA, H.; QUAGGIO, J.A.; FURLANI, A.M.C. Recomendações de adubação e calagem para o Estado de São Paulo. 2.ed. Campinas: Instituto Agronômico/Fundação IAC, 1997. p.175. (Boletim Técnico, 100)

VIGIER, B.; CUTCLIFFE, J.A. Effect of boron and nitrogen on the incidence of hollow stem in broccoli. Acta Horticulturae, v.157, p.303-308, 1984. 